\title{
The Follow-Up Investigation of Education Quality for Graduate in Applied Universities
}

\author{
Rong Chen \\ School of Physics and Electronic Information, Nanchang Normal University, Nanchang, China \\ Email: xinseafank@163.com
}

How to cite this paper: Chen, R. (2021) The Follow-Up Investigation of Education Quality for Graduate in Applied Universities. Open Access Library Journal, 8: e7981. https://doi.org/10.4236/oalib.1107981

Received: September 19, 2021

Accepted: October 15, 2021

Published: October 18, 2021

Copyright $\odot 2021$ by author(s) and Open Access Library Inc.

This work is licensed under the Creative Commons Attribution International License (CC BY 4.0).

http://creativecommons.org/licenses/by/4.0/

\section{(c) (i) Open Access}

\begin{abstract}
A questionnaire survey was conducted among graduates majoring in physics and electronic information engineering. This paper analyzes the distribution of employment industry, job satisfaction, professional counterpart, employment service, talent training and employment quality. It is found that there are deficiencies in the training of employment guidance and teaching practice. The results show that the follow-up investigation of the education quality for the graduate is of great significance for talent training. It provides a theoretical basis for promoting the reform of talent training mode and the revision of the training plan. It puts forward some countermeasures and suggestions to further improve and improve the education quality for graduates.
\end{abstract}

\section{Subject Areas}

Higher Education

\section{Keywords}

Graduates, Education Quality, Follow-Up Investigation

\section{Introduction}

In order to fully understand the education quality, employment status and development of graduates, further improve the talent education scheme and improve the education quality for graduates, a follow-up investigation on education quality of graduates is carried out in the form of a questionnaire survey. The questionnaire website is

https://www.wjx.cn/wjx/design/previewmobile.aspx?activity=116299734\&s=1.

It can enlighten students' employment guidance and guide them to establish a correct outlook on employment, further promote the reform of talent training, so as to extend the experience to the revision and optimization of talent educa- 
tion scheme, continue to improve the talent training system, make good plans for students' career, and provide scientific guidance for students' employment. Therefore, the follow-up investigation of graduates' education quality has important practical significance.

\section{Basic Information of Investigation}

The respondents of this survey are the 2020 undergraduate graduates of electronic information engineering and physics. The number of 2020 undergraduate graduates of electronic information engineering and physics is 105 . A total of 96 valid questionnaires were collected, including 55 males, accounting for $57.29 \%$ and 41 females, accounting for $42.71 \%$. Among the students, 13 are from cities, accounting for $13.54 \%, 20$ are from counties, accounting for $20.83 \%$, and 63 are from villages and towns, accounting for $65.63 \%$. 47 students majored in electronic information engineering, accounting for $48.96 \%$, and 49 students majored in physics, accounting for $51.04 \%$. There are 58 people working in Jiangxi Province, accounting for $60.42 \%$, and 38 people outside Jiangxi Province, accounting for $39.58 \%$. There are 5 students with a good family economy, accounting for $5.21 \%, 55$ students in general, accounting for $57.29 \%$, and 36 students poor, accounting for $37.5 \%$, as shown in Table 1 .

\section{Employment Analysis}

It can be seen from Table 2 that 31 people majoring in physics are engaged in education, accounting for $63.27 \%$, and 18 people engaged in other (Entrepreneurship), accounting for $36.73 \% .27$ students majoring in electronic information engineering continued to study and work in enterprises, accounting for $57.44 \%$, and 17 students majoring in other (Entrepreneurship), accounting for $36.17 \%$. From the perspective of the work industry, it is highly consistent with the specialty.

\section{Employment Services}

It can be seen from Table 3 that the employment services of physics are mainly

Table 1. Basic information of respondents.

\begin{tabular}{|c|c|c|c|c|c|c|c|}
\hline Question & Option & Freq. & Percentage & Question & Option & Freq. & Percentage \\
\hline \multirow[t]{2}{*}{ Sex } & Male & 55 & $57.29 \%$ & \multirow[t]{2}{*}{ Workplace } & $\begin{array}{l}\text { Jiangxi } \\
\text { Province }\end{array}$ & 58 & $60.42 \%$ \\
\hline & Female & 41 & $42.71 \%$ & & Other & 38 & $39.58 \%$ \\
\hline \multirow{3}{*}{ Hometown } & City & 13 & $13.54 \%$ & \multirow{3}{*}{$\begin{array}{c}\text { Family } \\
\text { affluence }\end{array}$} & Good & 5 & $5.21 \%$ \\
\hline & County & 20 & $20.83 \%$ & & General & 55 & $57.29 \%$ \\
\hline & Village and town & 63 & $65.63 \%$ & & Poor & 36 & $37.50 \%$ \\
\hline Major & $\begin{array}{l}\text { Electronic } \\
\text { information } \\
\text { engineering }\end{array}$ & 47 & $48.96 \%$ & Major & Physics & 49 & $51.04 \%$ \\
\hline
\end{tabular}


Table 2. Distribution of employment industries.

\begin{tabular}{ccc}
\hline Item & Physics & $\begin{array}{c}\text { Electronic information } \\
\text { engineering }\end{array}$ \\
\hline Further study (postgraduate, etc.) & $13(26.53 \%)$ & $15(31.91 \%)$ \\
Schools (kindergartens, primary and secondary schools) & $11(22.45 \%)$ & $0(0.00 \%)$ \\
Educational training institutions & $7(14.29 \%)$ & $1(2.13 \%)$ \\
Enterprises (private enterprises, \\
state-owned enterprises, foreign capital) \\
Civil servant & $0(0.00 \%)$ & $12(25.53 \%)$ \\
Others (Entrepreneurship) & $0(0.00 \%)$ & $2(4.26 \%)$ \\
Total & $18(36.73 \%)$ & $17(36.17 \%)$ \\
& 49 & 47 \\
\hline
\end{tabular}

Table 3. Employment services of physics.

\begin{tabular}{ccccc}
\hline $\begin{array}{c}\text { The school } \\
\text { organizes job fairs }\end{array}$ & $\begin{array}{c}\text { Career development } \\
\text { planning }\end{array}$ & $\begin{array}{c}\text { Publish recruitment } \\
\text { demand and salary information }\end{array}$ & $\begin{array}{c}\text { Job } \\
\text { counseling }\end{array}$ & Total \\
\hline $\begin{array}{c}24(48.98 \%) \\
\text { Coaching }\end{array}$ & Tutor resume writing & $20(40.82 \%)$ & $13(26.53 \%)$ & \\
$\begin{array}{c}\text { interview skills } \\
15(30.61 \%)\end{array}$ & $9(18.37 \%)$ & $4(8.16 \%)$ & $\begin{array}{c}\text { No job } \\
\text { counseling }\end{array}$ & 49 \\
& & & $5(10.20 \%)$ & \\
\hline
\end{tabular}

job fairs organized by the university (75.51\%), career development planning (48.98\%) and publishing recruitment information (40.82\%). It can be seen from Table 4 that the employment services of electronic information engineering are mainly job fairs organized by the university (76.6\%), recruitment information release (59.57\%) and career development planning (46.81\%). Students hope that the school will strengthen the release of employment information, on-site recruitment organization and career planning guidance. The school should provide more and high-quality employment information, optimize the organization of the recruitment site, including introducing high-quality enterprises, providing high-quality posts, and strengthening the guidance of students' career planning. Students are generally satisfied with the employment guidance and service of the school.

\section{Graduate Feedback}

It can be seen from Table 5 that students majoring in electronic information engineering have high evaluation on the talent training work of the school, and the top satisfaction is teachers' ethics, moral education, mental health education and teaching management level. It can be seen from Table 6 that students majoring in physics have high evaluation on the talent training work of the school, and the top satisfaction is teachers' ethics, moral education, mental health education, teaching management level, teaching teachers' level, school spirit and study style [1] [2] [3]. 
Table 4. Employment services of electronic information engineering.

\begin{tabular}{|c|c|c|c|c|}
\hline $\begin{array}{l}\text { The school } \\
\text { organizes job fairs }\end{array}$ & $\begin{array}{l}\text { Career development } \\
\text { planning }\end{array}$ & $\begin{array}{l}\text { Publish recruitment demand } \\
\text { and salary information }\end{array}$ & $\begin{array}{c}\text { Job } \\
\text { counseling }\end{array}$ & Total \\
\hline $36(76.60 \%)$ & $22(46.81 \%)$ & $28(59.57 \%)$ & $13(27.66 \%)$ & \\
\hline $\begin{array}{l}\text { Coaching } \\
\text { interview skills }\end{array}$ & Tutor resume writing & Recommended work & $\begin{array}{c}\text { No job } \\
\text { counseling }\end{array}$ & 47 \\
\hline $9(19.15 \%)$ & $6(12.77 \%)$ & $6(12.77 \%)$ & $5(10.64 \%)$ & \\
\hline
\end{tabular}

Table 5. Objective evaluation of students majoring in electronic information engineering on talent training of the University.

\begin{tabular}{|c|c|c|c|c|c|c|}
\hline Item & $\begin{array}{c}\text { Very } \\
\text { satisfied }\end{array}$ & Satisfied & Commonly & $\begin{array}{l}\text { Not very } \\
\text { satisfied }\end{array}$ & Dissatisfied & Total \\
\hline Talent training program & $12(25.53 \%)$ & $22(46.81 \%)$ & $13(27.66 \%)$ & $0(0.00 \%)$ & $0(0.00 \%)$ & 47 \\
\hline Overall curriculum system & $12(25.53 \%)$ & $21(44.68 \%)$ & $14(29.79 \%)$ & $0(0.00 \%)$ & $0(0.00 \%)$ & 47 \\
\hline $\begin{array}{l}\text { Coincidence between } \\
\text { core courses and work }\end{array}$ & $12(25.53 \%)$ & $20(42.55 \%)$ & $15(31.91 \%)$ & $0(0.00 \%)$ & $0(0.00 \%)$ & 47 \\
\hline Teaching methods & $12(25.53 \%)$ & $22(46.81 \%)$ & $13(27.66 \%)$ & $0(0.00 \%)$ & $0(0.00 \%)$ & 47 \\
\hline $\begin{array}{l}\text { Experimental } \\
\text { training conditions }\end{array}$ & $11(23.40 \%)$ & $21(44.68 \%)$ & $15(31.91 \%)$ & $0(0.00 \%)$ & $0(0.00 \%)$ & 47 \\
\hline Practice implementation & $12(25.53 \%)$ & $20(42.55 \%)$ & $15(31.91 \%)$ & $0(0.00 \%)$ & $0(0.00 \%)$ & 47 \\
\hline Teaching teacher level & $14(29.79 \%)$ & $23(48.94 \%)$ & $10(21.28 \%)$ & $0(0.00 \%)$ & $0(0.00 \%)$ & 47 \\
\hline Teachers' morality and style & $14(29.79 \%)$ & $24(51.06 \%)$ & $9(19.15 \%)$ & $0(0.00 \%)$ & $0(0.00 \%)$ & 47 \\
\hline Moral education & $16(34.04 \%)$ & $22(46.81 \%)$ & $9(19.15 \%)$ & $0(0.00 \%)$ & $0(0.00 \%)$ & 47 \\
\hline Mental health education & $15(31.91 \%)$ & $23(48.94 \%)$ & $9(19.15 \%)$ & $0(0.00 \%)$ & $0(0.00 \%)$ & 47 \\
\hline School spirit and study style & $16(34.04 \%)$ & $20(42.55 \%)$ & $11(23.40 \%)$ & $0(0.00 \%)$ & $0(0.00 \%)$ & 47 \\
\hline School running conditions & $15(31.91 \%)$ & $21(44.68 \%)$ & $11(23.40 \%)$ & $0(0.00 \%)$ & $0(0.00 \%)$ & 47 \\
\hline Teaching management level & $15(31.91 \%)$ & $22(46.81 \%)$ & $10(21.28 \%)$ & $0(0.00 \%)$ & $0(0.00 \%)$ & 47 \\
\hline Teaching facilities & $13(27.66 \%)$ & $23(48.94 \%)$ & $11(23.40 \%)$ & $0(0.00 \%)$ & $0(0.00 \%)$ & 47 \\
\hline
\end{tabular}

Table 6. Objective evaluation of students majoring in physics on talent training in the school.

\begin{tabular}{ccccccc}
\hline Item & $\begin{array}{c}\text { Very } \\
\text { satisfied }\end{array}$ & Satisfied & Commonly & $\begin{array}{c}\text { Not very } \\
\text { satisfied }\end{array}$ & Dissatisfied Total \\
\hline $\begin{array}{c}\text { Talent training program } \\
\text { Overall curriculum system }\end{array}$ & $12(24.49 \%)$ & $18(36.73 \%)$ & $17(34.69 \%)$ & $2(4.08 \%)$ & $0(0.00 \%)$ & 49 \\
$\begin{array}{c}\text { Coincidence between } \\
\text { core courses and work } \\
\text { Teaching methods }\end{array}$ & $12(24.49 \%)$ & $21(42.86 \%)$ & $15(30.61 \%)$ & $1(2.04 \%)$ & $0(0.00 \%)$ & 49 \\
$\begin{array}{c}\text { Experimental } \\
\text { training conditions }\end{array}$ & $13(26.53 \%)$ & $18(36.73 \%)$ & $18(36.73 \%)$ & $0(0.00 \%)$ & $0(0.00 \%)$ & 49 \\
$\begin{array}{c}\text { Practice implementation } \\
\text { Teaching teacher level }\end{array}$ & $13(26.53 \%)$ & $19(38.78 \%)$ & $16(32.65 \%)$ & $1(2.04 \%)$ & $0(0.00 \%)$ & 49 \\
\hline
\end{tabular}




\section{Continued}

\begin{tabular}{|c|c|c|c|c|}
\hline Teachers' morality and style & $17(34.69 \%) 22(44.90 \%) 10(20.41 \%)$ & $0(0.00 \%)$ & $0(0.00 \%)$ & 49 \\
\hline Moral education & $14(28.57 \%) 21(42.86 \%) 14(28.57 \%)$ & $0(0.00 \%)$ & $0(0.00 \%)$ & 49 \\
\hline Mental health education & $15(30.61 \%) 20(40.82 \%) 14(28.57 \%)$ & $0(0.00 \%)$ & $0(0.00 \%)$ & 49 \\
\hline School spirit and study style & $14(28.57 \%) 20(40.82 \%) 15(30.61 \%)$ & $0(0.00 \%)$ & $0(0.00 \%)$ & 49 \\
\hline School running conditions & $13(26.53 \%) 20(40.82 \%) 16(32.65 \%)$ & $0(0.00 \%)$ & $0(0.00 \%)$ & 49 \\
\hline Teaching management level & $14(28.57 \%) 20(40.82 \%) 15(30.61 \%)$ & $0(0.00 \%)$ & $0(0.00 \%)$ & 49 \\
\hline Teaching facilities & $14(28.57 \%) 19(38.78 \%) 16(32.65 \%)$ & $0(0.00 \%)$ & $0(0.00 \%)$ & 49 \\
\hline
\end{tabular}

It can be seen from Table 7 that students majoring in physics think that the most important quality is ability quality, and students majoring in electronic information engineering think that the most important quality is psychological quality. It can be seen from Table 8 that physics believe that the most important ability is professional skills, while electronic information engineering students believe that the most important ability is practical ability.

It can be seen from Table 9 that $70 \%$ of the students majoring in electronic information engineering believe that their professional skills are obtained through the talent training of our university, and $50 \%$ of the students believe that their other abilities are also obtained through the talent training of our university. It can be seen from Table 10 that $70 \%$ of the students majoring in physics believe that their professional skills are obtained through the talent training of our university, and $50 \%$ of the students believe that their other abilities are also obtained through the talent training of our university.

It can be seen from Table 11 that during school, nearly $70 \%$ of students believe that the teachers who have the greatest impact on students' education are professional teachers, whether physics or electronic information engineering. Table 12 shows that during school, nearly $70 \%$ of students, whether physics or electronic information engineering, believe that the biggest teaching link for the improvement and development of students' ability is experimental training teaching, followed by internship. It can be seen from Table 13 that $70 \%$ of physics students think that the time arrangement of experimental training and practice is more appropriate. Fifty percent of electronic information engineering students think that the time arrangement of experimental training and practice is more appropriate. As can be seen from Table 14, whether physics or electronic information engineering, students believe that the key factor affecting the teaching quality is the talent training mode, followed by the level of school teachers. It can be seen from Table 15 that students majoring in physics believe that the work that the school needs to strengthen in talent training is professional basic knowledge and organization and coordination ability. Students majoring in electronic information engineering should first strengthen their practical ability, followed by professional basic knowledge. It can be seen from Table 16 that more than $70 \%$ of the students majoring in physics or electronic information engineering are generally satisfied with the school's education and teaching work. 
Table 7. Your most important qualities.

\begin{tabular}{cccccccc}
\hline Major & $\begin{array}{c}\text { Physical } \\
\text { quality }\end{array}$ & $\begin{array}{c}\text { Psychological } \\
\text { quality }\end{array}$ & $\begin{array}{c}\text { Ideological and } \\
\text { political quality }\end{array}$ & $\begin{array}{c}\text { Moral } \\
\text { quality }\end{array}$ & $\begin{array}{c}\text { Knowledge } \\
\text { quality }\end{array}$ & $\begin{array}{c}\text { Ability and } \\
\text { quality }\end{array}$ & $\begin{array}{c}\text { Total } \\
\text { Physics }\end{array}$ \\
$6(12.24 \%)$ & $10(20.41 \%)$ & $5(10.20 \%)$ & $8(16.33 \%)$ & $8(16.33 \%)$ & $12(24.49 \%)$ & 49 \\
\hline $\begin{array}{c}\text { Electronic information } \\
\text { engineering }\end{array}$ & $9(19.15 \%)$ & $15(31.91 \%)$ & $5(10.64 \%)$ & $6(12.77 \%)$ & $2(4.26 \%)$ & $10(21.28 \%)$ & 47 \\
\hline
\end{tabular}

Table 8. Your most important competencies.

\begin{tabular}{|c|c|c|c|c|c|c|c|c|}
\hline Major & $\begin{array}{l}\text { Professional } \\
\text { skills }\end{array}$ & $\begin{array}{c}\text { Language } \\
\text { expression ability }\end{array}$ & $\begin{array}{l}\text { Organization and } \\
\text { coordination ability }\end{array}$ & $\begin{array}{l}\text { Practical } \\
\text { ability }\end{array}$ & $\begin{array}{l}\text { Innovation } \\
\text { ability }\end{array}$ & $\begin{array}{l}\text { Social } \\
\text { ability }\end{array}$ & $\begin{array}{l}\text { Self control } \\
\text { ability }\end{array}$ & Total \\
\hline Physics & $22(44.90 \%)$ & $9(18.37 \%)$ & $6(12.24 \%)$ & $4(8.16 \%)$ & $2(4.08 \%)$ & $4(8.16 \%)$ & $2(4.08 \%)$ & 49 \\
\hline $\begin{array}{c}\text { Electronic information } \\
\text { engineering }\end{array}$ & $14(29.79 \%)$ & $9(19.15 \%)$ & $5(10.64 \%)$ & $15(31.91 \%)$ & $0(0.00 \%)$ & $3(6.38 \%)$ & $1(2.13 \%)$ & 47 \\
\hline
\end{tabular}

Table 9. The ability of students majoring in electronic information engineering obtained through talent training in our university.

\begin{tabular}{ccccccc}
\hline Item & Entire & Most & Half & $\begin{array}{c}\text { Smaller } \\
\text { part }\end{array}$ & $\begin{array}{c}\text { Not a } \\
\text { quarter }\end{array}$ & Total \\
\hline Professional skills & $8(17.02 \%)$ & $25(53.19 \%)$ & $12(25.53 \%)$ & $2(4.26 \%)$ & $0(0.00 \%)$ & 47 \\
$\begin{array}{c}\text { Language } \\
\text { expression ability }\end{array}$ & $6(12.77 \%)$ & $22(46.81 \%)$ & $16(34.04 \%)$ & $3(6.38 \%)$ & $0(0.00 \%)$ & 47 \\
$\begin{array}{c}\text { Organization and } \\
\text { coordination ability }\end{array}$ & $6(12.77 \%)$ & $21(44.68 \%)$ & $19(40.43 \%)$ & $1(2.13 \%)$ & $0(0.00 \%)$ & 47 \\
Practical ability & $6(12.77 \%)$ & $26(55.32 \%)$ & $14(29.79 \%)$ & $1(2.13 \%)$ & $0(0.00 \%)$ & 47 \\
$\begin{array}{c}\text { Innovation ability } \\
\text { Social ability }\end{array}$ & $7(14.89 \%)$ & $21(44.68 \%)$ & $17(36.17 \%)$ & $2(4.26 \%)$ & $0(0.00 \%)$ & 47 \\
Self control ability & $6(12.77 \%)$ & $18(38.30 \%)$ & $21(44.68 \%)$ & $2(4.26 \%)$ & $0(0.00 \%)$ & 47 \\
\hline
\end{tabular}

Table 10. The ability of students majoring in physics obtained through talent training in our university.

\begin{tabular}{ccccccc}
\hline Item & Entire & Most & Half & $\begin{array}{c}\text { Smaller } \\
\text { part }\end{array}$ & $\begin{array}{c}\text { Nota } \\
\text { quarter }\end{array}$ & Total \\
\hline $\begin{array}{c}\text { Professional skills } \\
\text { Language }\end{array}$ & $11(22.45 \%)$ & $24(48.98 \%)$ & $14(28.57 \%)$ & $0(0.00 \%)$ & $0(0.00 \%)$ & 49 \\
$\begin{array}{c}\text { expression ability } \\
\text { Organization and } \\
\text { coordination ability }\end{array}$ & $9(16.33 \%)$ & $22(44.90 \%)$ & $15(30.61 \%)$ & $4(8.16 \%)$ & $0(0.00 \%)$ & 49 \\
$\begin{array}{c}\text { Practical ability } \\
\text { Innovation ability }\end{array}$ & $10(20.41 \%)$ & $21(42.86 \%)$ & $16(32.65 \%)$ & $2(4.08 \%)$ & $0(0.00 \%)$ & 49 \\
Social ability & $8(16.33 \%)$ & $22(44.90 \%)$ & $16(32.65 \%)$ & $2(4.08 \%)$ & $1(2.04 \%)$ & 49 \\
Self control ability & $7(14.29 \%)$ & $22(44.90 \%)$ & $18(36.73 \%)$ & $1(2.04 \%)$ & $1(2.04 \%)$ & 49 \\
\hline
\end{tabular}


Table 11. Teachers who have the greatest impact on your education during school.

\begin{tabular}{cccccc}
\hline Major & $\begin{array}{c}\text { Speciality } \\
\text { teachers }\end{array}$ & $\begin{array}{c}\text { Basic } \\
\text { Course }\end{array}$ & Counselor & $\begin{array}{c}\text { Other } \\
\text { teachers }\end{array}$ & Total \\
\hline Physics & $33(67.35 \%)$ & $4(8.16 \%)$ & $7(14.29 \%)$ & $5(10.20 \%)$ & 49 \\
$\begin{array}{c}\text { Electronic } \\
\text { information engineering }\end{array}$ & $32(68.09 \%)$ & $1(2.13 \%)$ & $5(10.64 \%)$ & $9(19.15 \%)$ & 47 \\
\hline
\end{tabular}

Table 12. What teaching links do you think are the most important to the improvement and development of students' ability during school.

\begin{tabular}{cccccc}
\hline Major & $\begin{array}{c}\text { Theory } \\
\text { Teaching }\end{array}$ & $\begin{array}{c}\text { Experimental } \\
\text { training } \\
\text { teaching }\end{array}$ & $\begin{array}{c}\text { Graduation } \\
\text { thesis }\end{array}$ & $\begin{array}{c}\text { Educational } \\
\text { practice or } \\
\text { engineering practice }\end{array}$ & Total \\
\hline $\begin{array}{c}\text { Physics } \\
\text { Electronic }\end{array}$ & $9(18.37 \%)$ & $16(32.65 \%)$ & $3(6.12 \%)$ & $21(42.86 \%)$ & 49 \\
enformation & $7(14.89 \%)$ & $25(53.19 \%)$ & $5(10.64 \%)$ & $10(21.28 \%)$ & 47 \\
\hline
\end{tabular}

Table 13. Arrangement of students' experimental training and practice time during school.

\begin{tabular}{|c|c|c|c|c|c|c|}
\hline Major & $\begin{array}{c}\text { Very } \\
\text { appropriate }\end{array}$ & $\begin{array}{c}\text { More } \\
\text { appropriate }\end{array}$ & Commonly & $\begin{array}{c}\text { More } \\
\text { inappropriate }\end{array}$ & $\begin{array}{c}\text { Very } \\
\text { inappropriate }\end{array}$ & Total \\
\hline Physics & $11(22.45 \%)$ & $24(48.98 \%)$ & $12(24.49 \%)$ & $2(4.08 \%)$ & $0(0.00 \%)$ & 49 \\
\hline $\begin{array}{l}\text { Electronic } \\
\text { information } \\
\text { engineering }\end{array}$ & $10(21.28 \%)$ & $12(25.53 \%)$ & $19(40.43 \%)$ & $3(6.38 \%)$ & $3(6.38 \%)$ & 47 \\
\hline
\end{tabular}

Table 14. Do you think what are the key factors affecting teaching quality.

\begin{tabular}{ccccccc}
\hline Major & $\begin{array}{c}\text { Teachers' } \\
\text { level }\end{array}$ & $\begin{array}{c}\text { Talent } \\
\text { training } \\
\text { mode }\end{array}$ & $\begin{array}{c}\text { Quality of } \\
\text { new students }\end{array}$ & $\begin{array}{c}\text { Teaching } \\
\text { management } \\
\text { level }\end{array}$ & Other & Total \\
\hline Physics & $13(26.53 \%)$ & $16(32.65 \%)$ & $5(10.20 \%)$ & $11(22.45 \%)$ & $4(8.16 \%)$ & 49 \\
$\begin{array}{c}\text { Electronic } \\
\text { information } \\
\text { engineering }\end{array}$ & $12(25.53 \%)$ & $25(53.19 \%)$ & $2(4.26 \%)$ & $5(10.64 \%)$ & $3(6.38 \%)$ & 47 \\
\hline
\end{tabular}

Table 15. What do you think the school needs to strengthen in talent training.

\begin{tabular}{|c|c|c|c|c|c|c|}
\hline & $\begin{array}{l}\text { Professional } \\
\text { basic } \\
\text { knowledge }\end{array}$ & $\begin{array}{c}\text { Ideological } \\
\text { and political } \\
\text { quality }\end{array}$ & $\begin{array}{c}\text { Practical } \\
\text { ability }\end{array}$ & $\begin{array}{c}\text { Faculty for } \\
\text { adapting } \\
\text { environments }\end{array}$ & $\begin{array}{c}\text { Organization } \\
\text { and } \\
\text { coordination } \\
\text { ability }\end{array}$ & Total \\
\hline \multirow[t]{3}{*}{ Physics } & $29(59.18 \%)$ & $21(42.86 \%)$ & $30(61.22 \%)$ & $15(30.61 \%)$ & $24(48.98 \%)$ & \\
\hline & $\begin{array}{c}\text { Language } \\
\text { expression } \\
\text { ability }\end{array}$ & $\begin{array}{l}\text { Problem } \\
\text { solving } \\
\text { ability }\end{array}$ & $\begin{array}{l}\text { Foreign language } \\
\text { and computer } \\
\text { application ability }\end{array}$ & $\begin{array}{c}\text { Creative } \\
\text { consciousness } \\
\text { and ability }\end{array}$ & Other & 49 \\
\hline & $23(46.94 \%)$ & $13(26.53 \%)$ & $12(24.49 \%)$ & $16(32.65 \%)$ & $6(12.24 \%)$ & \\
\hline
\end{tabular}




\begin{tabular}{ccccccc} 
Continued & \multicolumn{1}{c}{$\begin{array}{l}\text { Professional } \\
\text { basic } \\
\text { knowledge }\end{array}$} & $\begin{array}{c}\text { Ideological } \\
\text { and political } \\
\text { quality }\end{array}$ & Practical ability & $\begin{array}{c}\text { Faculty for } \\
\text { adapting } \\
\text { environments }\end{array}$ & $\begin{array}{c}\text { Organization } \\
\text { and } \\
\text { coordination } \\
\text { ability }\end{array}$ & Total \\
$\begin{array}{c}\text { Electronic } \\
\text { information } \\
\text { engineering }\end{array}$ & $\begin{array}{c}28(59.57 \%) \\
\text { Language } \\
\text { expression } \\
\text { ability }\end{array}$ & $\begin{array}{c}\text { Problem } \\
\text { solving ability }\end{array}$ & $\begin{array}{c}\text { Foreign language } \\
\text { and computer } \\
\text { application ability }\end{array}$ & $\begin{array}{c}\text { Creative } \\
\text { consciousness } \\
\text { and ability }\end{array}$ & Other & 47 \\
$19(40.43 \%)$ & $17(36.17 \%)$ & $10(21.28 \%)$ & $18(38.30 \%)$ & $4(8.51 \%)$ & \\
\hline
\end{tabular}

Table 16. Satisfaction of school education and teaching.

\begin{tabular}{ccccccc}
\hline Major & Very satisfied & Satisfied & Commonly & Not very satisfied & Dissatisfied & Total \\
\hline Physics & $12(24.49 \%)$ & $23(46.94 \%)$ & $14(28.57 \%)$ & $0(0.00 \%)$ & $0(0.00 \%)$ & 49 \\
$\begin{array}{c}\text { Electronic } \\
\text { information } \\
\text { engineering }\end{array}$ & $11(23.40 \%)$ & $26(55.32 \%)$ & $10(21.28 \%)$ & $0(0.00 \%)$ & $0(0.00 \%)$ & 47 \\
\hline
\end{tabular}

\section{Problems in Talent Training}

1) Electronic information engineering and physics have high requirements for experimental training. In view of the constraints of the experimental training course by the objective conditions such as insufficient experimental equipment and insufficient experimental site, what's more, the experimental training course should be divided into groups, resulting in the inappropriate arrangement of experimental training time.

2) The practical teaching of experimental training should present a low-level, medium-level and high-level progressive type, and the talent training scheme should be optimized. The practical teaching should start from the lower grade students and be arranged as early as possible, so as to have the time and energy to engage in progressive teaching.

3) As science and engineering majors have high requirements for the practical ability of teachers, and the practical ability of teachers needs to be further improved.

\section{Improvement Measures and Suggestions on Professional Talent Training}

1) Relevant professional teachers are required to enter the enterprise for temporary training. Promote the integration of industry and education, schools and enterprises should cooperate deeply, send teachers to enterprises to carry out temporary training, and introduce industry experts to guide professional teaching. At the same time, teachers should also assist enterprises in professional training or undertake certain scientific research work, help enterprise employees improve their teaching level, enhance enterprise influence and service quality.

2) The training base is an important place for training course teaching and an 
important guarantee for talent training. With the help of school enterprise cooperation and the application of various professional training bases at all levels, strengthen the construction of training bases, make the training bases inside and outside the school from specialized to refined, keep pace with the times, and reflect the scientificity, demonstration and guidance of the construction of training bases.

3) Enhance employ-ability

Through the follow-up survey of graduates, fully understand the needs of employers and students, build a better curriculum system, guide the reform of curriculum content, integrate the cultivation of humanistic quality into the professional knowledge system, reform the classroom teaching organization, build a good learning atmosphere and learning environment, create a better and better employment environment for school graduates and improve their employ-ability.

\section{Application of Survey Results in Talent Training Program Formulation and Talent Training}

1) Society and market demand determine the training objectives and positioning of professional students. It is unrealistic to talk about the reform of talent training program without industry. We should strengthen students' professional cognition, focus on cultivating students' professional consciousness and strengthen students' daily behavior management. Therefore, professional personnel training should be in line with the industry, and teaching standards and student management should be connected with industry management standards. The focus of talent training should not only be on the cultivation of professional skills, but also on the cultivation of professional identity, professional quality, professional ethics, service consciousness, language communication and cultural and artistic aesthetics. Education and teaching should not be limited to books and classrooms, but also make full use of information resources, combine inside and outside the classroom and inside and outside the school, give full play to the role of associations and the second classroom, and show the professional talent training concept by organizing students to participate in various activities, inviting excellent graduates and experts to the school to carry out lectures and exchange activities.

2) The follow-up survey results of graduates can reflect the quality of professional talent training, whether the ideological quality, professional ability and professional knowledge of graduates can meet the needs of employers, and whether they can adapt to the pace of regional economic development is related to the sustainable development of the major. Therefore, the follow-up survey of graduates can promote the construction of the major, enhance professional competitiveness and the ability to serve the regional economy.

\section{Discussion}

The sample size of this study is insufficient, and it will be improved in the future. 
The samples are not very representative, and typical samples will be selected in the future.

\section{Acknowledgements}

Project supported by the research on teaching reform of Nanchang Normal University (Grant No. JGKT-19-12) and also by Training program for college students (Grant No. 201914437012, 201914437013). With funding, we can better carry out teaching research and apply the research results to teaching, so as to improve the quality of teaching.

\section{Conflicts of Interest}

The author declares no conflicts of interest.

\section{References}

[1] Warren, L.L. (2021) The Importance of Teacher Leadership Skills in the Classroom. Education Journal, 10, 8-15. https://doi.org/10.11648/j.edu.20211001.12

[2] Machuca, J.M.C. et al. (2021) Educational Leadership as the Impact of Teachers in the Formation of the Attitude of Students from a Manichean Perspective. Education Journal, 10, 16-22. https://doi.org/10.11648/j.edu.20211001.13

[3] Somano, T.T. and Ololo, Y.F. (2021) Enhancing Active Learning Methods in Department of Physics Students in Wolaita Sodo University. Higher Education Research, 6, 86-92. 